\title{
Manifolds with weighted Poincaré inequality and uniqueness of minimal hypersurfaces
}

\author{
Xu Cheng and Detang Zhou
}

In this paper, we obtain results on rigidity of complete Riemannian manifolds with weighted Poincaré inequality. As an application, we prove that if $M$ is a complete $\frac{n-2}{n}$-stable minimal hypersurface in $\mathbb{R}^{n+1}$ with $n \geq 3$ and has bounded norm of the second fundamental form, then $M$ must either have only one end or be a catenoid.

\section{Introduction}

In this paper, we will discuss complete Riemannian manifolds with weighted Poincaré inequality and minimal hypersurfaces with $\delta$-stability in the Euclidian space $\mathbb{R}^{n+1}$ with $n \geq 3$. We first recall some backgrounds.

Let $M$ be an $n$-dimensional Riemannian manifold. Given a Schrödinger operator $L=\Delta+q(x)$ on $M$, we consider the eigenvalue problem on a compact subdomain $D \subset M$ :

$$
\left\{\begin{array}{l}
L f+\lambda f=0, \quad \text { in } D \\
\left.f\right|_{\partial D}=0 .
\end{array}\right.
$$

It has discrete spectrum and the number of negative eigenvalues is finite. The (Morse) index of $\mathrm{L}$ on $M$ is defined as the supremum, over compact domains of $M$, of the number of negative eigenvalues (counted with multiplicity) of $L$ with Dirichlet boundary condition.

If $M$ is a complete connected immersed minimal hypersurface in $\mathbb{R}^{n+1}$, $n \geq 2$ and if $L$ is the Jacobi operator $L=\Delta+|A|^{2}$, where $|A|$ denotes the norm of the second fundamental form of $M$, then the index of $L$ is said to be the (Morse) index of $M . M$ is said to be stable if the index of $M$ is 0 , which is equivalent to say that, for all compactly supported piecewise 
smooth function $f \in C_{o}^{\infty}(M)$,

$$
\int_{M}\left(|\nabla f|^{2}-|A|^{2} f^{2}\right) \geq 0 .
$$

It is known that a complete stable minimal surface in $\mathbb{R}^{3}$ is plane, which was proved by do Carmo and Peng and Fischer-Cobrie and Schoen independently $[6,7]$; and that only index one complete minimal surfaces in $\mathbb{R}^{3}$ are the catenoid and Enneper surface, which was proved by Lopez and Ros [9].

While it is unknown that a complete stable minimal hypersurface in $\mathbb{R}^{n+1}$ is a hyperplane when $n \leq 7$, Cao et al. [1] proved that a complete stable minimal hypersurface in $\mathbb{R}^{n+1}$ must have only one end for all dimension $n \geq 3$. Tam and Zhou [16] recently showed that an ( $n$-dimensional) catenoid in the Euclidean space $\mathbb{R}^{n+1}$ with $n \geq 3$ has index one (see the definition of $n$-dimensional catenoid in $\mathbb{R}^{n+1}$ in [5], also in [16]).

Now let us assume $L=\Delta+\delta|A|^{2}$ on minimal hypersurface $M$ in $\mathbb{R}^{n+1}$ for some number $0<\delta \leq 1$. We may similarly define that $M$ is $\delta$-stable if

$$
\int_{M}\left(|\nabla f|^{2}-\delta|A|^{2} f^{2}\right) \geq 0
$$

for all $f \in C_{o}^{\infty}(M)$.

Obviously, given $\delta_{1}>\delta_{2}, \delta_{1}$-stable implies $\delta_{2}$-stable. So $M$ is stable implies that $M$ is $\delta$-stable for all $0<\delta \leq 1$. Hyperplane is $\delta$-stable for all $0<\delta \leq 1$.

There are some work on $\delta$-stable minimal hypersurfacs. Kawai [8] proved a $\delta$-stable, $\delta>\frac{1}{8}$ complete minimal surface in $\mathbb{R}^{3}$ must be plane. Recently, Meeks et al. [14] showed that any complete embedded $\delta$-stable minimal surface in $\mathbb{R}^{3}$ with finite genus is flat. In the case of higher dimension $n \geq 3$, we have, directly from the argument in [1], that the result of Cao, Shen and Zhu also holds for $\frac{n-1}{n}$-stable. Recently, Tam and Zhou [16] showed that a catenoid in $\mathbb{R}^{n+1}$ is $\frac{n-2}{n}$-stable. Also they proved that if $M$ is an $\frac{n-2}{n}$-stable complete immersed minimal hypersurface in $\mathbb{R}^{n+1}$ and if

$$
\lim _{R \rightarrow+\infty} \frac{1}{R^{2}} \int_{B(2 R) \backslash B(R)}|A|^{2(n-2) / n}=0,
$$

then $M$ is either a hyperplane or a catenoid.

In this paper, we prove that if an $\frac{n-2}{n}$-stable complete minimal hypersurface in $\mathbb{R}^{n+1}$ with $n \geq 3$ and the norm of its second fundamental form satisfies some growth condition, then it either has only one end or is a catenoid. More precisely, we show 
Weighted Poincaré inequality and uniqueness of minimal hypersurfaces 141

Theorem 1.1. Let $M$ be an $\frac{n-2}{n}$-stable complete minimal hypersurface in $\mathbb{R}^{n+1}$ for $n \geq 3$ and the norm of its second fundamental form satisfies

$$
\begin{aligned}
& \lim _{R \rightarrow+\infty} \sup _{B(R)}|A| / R^{(n-3) / 2}=0 \quad \text { for } \quad n \geq 4 ; \\
& \lim _{R \rightarrow+\infty} \sup _{B(R)}|A| / \ln R=0 \quad \text { for } \quad n=3,
\end{aligned}
$$

then $M$ either has only one end or is a catenoid.

From Theorem 1.1, we have the following result:

Corollary 1.1. Let $M$ be an $\frac{n-2}{n}$-stable complete minimal hypersurface in $\mathbb{R}^{n+1}, n \geq 3$, with at least two ends. If it has bounded norm of the second fundamental form, then $M$ must be a catenoid.

Our results for minimal hypersurfaces rely on the study on complete manifolds with weighted Poincaré inequality which is of independent interest.

Let $M$ be a complete Riemannian manifold. Recall from [13] that a complete Riemannian manifold $\left(M, d s^{2}\right)$ is said to satisfy a weighted Poincaré inequality with non-negative weight function $\rho$ if the inequality

$$
\int_{M}|\nabla f|^{2} \geq \int_{M} \rho f^{2}
$$

holds for all compactly supported piecewise smooth function $f \in C_{o}^{+\infty}(M)$.

Further, $M$ is said to satisfy property $\left(\mathcal{P}_{\rho}\right)$ for non-zero non-negative weight function $\rho(x)$ if,

(1) $M$ satisfies a weighted Poincaré inequality with $\rho$; and

(2) the conformal metric $\rho d s^{2}$ is complete.

In [13], Li and Wang studied complete manifolds satisfying property $\left(\mathcal{P}_{\rho}\right)$ and obtained many theorems on rigidity. Later the first author [3] discussed complete manifolds with Poincaré inequality and obtain results on the uniqueness of ends which can be applied to study stable minimal hypersurfaces in a Riemannian manifold. In this paper, we generalize one result of Li and Wang [13, Theorem 5.2] to the following:

Theorem 1.2. Let $M$ be a complete $n$-dimensional $(n \geq 3)$ Riemannian manifold with property $\left(\mathcal{P}_{\rho}\right)$ for some non-zero weight function $\rho$. Suppose 
the Ricci curvature of $M$ has the lower bound

$$
\operatorname{Ric}_{M}(x) \geq-(n-1) \tau(x), \quad x \in M,
$$

where $\tau(x)$ satisfies Poincaré inequality

$$
\int_{M}|\nabla f|^{2} \geq(n-2) \int_{M} \tau f^{2}, \text { for all } f \in C_{o}^{+\infty}(M) .
$$

If $\rho$ and $\tau$ satisfy the growth condition

$$
\begin{aligned}
& \liminf _{R \rightarrow+\infty} S(R) e^{-((n-3) /(n-2)) R}=0 \quad \text { for } \quad n \geq 4, \\
& \liminf _{R \rightarrow+\infty} S(R) R^{-1}=0 \quad \text { for } \quad n=3
\end{aligned}
$$

where

$$
S(R)=\sup _{x \in B_{\rho}(R)}(\sqrt{\rho(x)}, \sqrt{\tau(x)})
$$

then either

(1) $M$ has only one non-parabolic end; or

(2) $M$ has two non-parabolic ends and is given by $M=\mathbb{R} \times N$ with the warped product metric

$$
d s_{M}^{2}=d t^{2}+\eta^{2}(t) d s_{N}^{2},
$$

for some positive function $\eta(t)$ and some compact manifold $N$. Moreover, $\tau(t)$ is a function of $t$ alone satisfying

$$
(n-2) \eta^{\prime \prime} \eta^{-1}=\tau \text {. }
$$

If we choose $\tau=\frac{1}{n-2} \rho$ in Theorem 1.2, it is just Theorem 5.2 of [13]. In the case of minimal hypersurfaces, we could not find any weight function $\rho$ in a Poincaré inequality, which satisfies both the completeness of the metric $\rho d s^{2}$ and the lower bound estimate of Ricci curvature of $M$. Hence, we could not apply the theorem of Li and Wang. Instead, our Theorem 1.2 is suitable to our minimal case (see Theorem 1.1).

The work of $\mathrm{Li}$ and Wang on complete manifolds satisfying weighted Poincaré inequality is a generalization of their one on complete manifolds with positive spectrum [11,12]; see [13] and the references therein). Let $\lambda_{1}(M)$ be the largest lower bound of the spectrum of the Laplacian with respect to the metric of $M$. Theorem 1.2 implies the following result. 
Corollary 1.2. Let $M$ be a complete $n$-dimensional $(n \geq 3)$ Riemannian manifold with positive spectrum (i.e., $\left.\lambda_{1}(M)>0\right)$. Suppose the Ricci curvature of $M$ has the lower bound

$$
\operatorname{Ric}_{M}(x) \geq-(n-1) \tau(x), \quad x \in M
$$

where $\tau(x)$ satisfies Poincaré inequality

$$
\int_{M}|\nabla f|^{2} \geq(n-2) \int_{M} \tau f^{2}, \text { for all } f \in C_{o}^{+\infty}(M) .
$$

If $\tau$ satisfies the growth condition

$$
\begin{aligned}
& \liminf _{R \rightarrow+\infty}\left(\sup _{x \in B(R)} \tau(x)\right) e^{-(2(n-3) /(n-2)) R}=0 \quad \text { for } n \geq 4 \\
& \liminf _{R \rightarrow+\infty}\left(\sup _{x \in B(R)} \tau(x)\right) R^{-2}=0 \quad \text { for } \quad n=3
\end{aligned}
$$

then either

(1) $M$ has only one non-parabolic end; or

(2) $M$ has two non-parabolic ends and is given by $M=\mathbb{R} \times N$ with the warped product metric

$$
d s_{M}^{2}=d t^{2}+\eta^{2}(t) d s_{N}^{2}
$$

for some positive function $\eta(t)$ and some compact manifold $N$. Moreover, $\tau(t)$ is a function of $t$ alone satisfying

$$
(n-2) \eta^{\prime \prime} \eta^{-1}=\tau \text {. }
$$

This corollary generalizes Theorem 2.1 in [11] (just choose $\tau(x)=\frac{\lambda_{1}(M)}{n-2}$ and use the fact a non-parabolic end with $\lambda_{1}(M)>0$ has infinite volume).

Throughout this paper, all manifolds are assumed to be oriented.

\section{Rigidity of complete manifolds}

In this section, we will consider the structure of a complete manifold $M$ with property $\left(\mathcal{P}_{\rho}\right)$. Since we follow the argument of Li and Wang [13, Theorem 5.2 ] with some changes of techniques in the proof of our Theorem 1.2, we recommend [13] as a complement when necessary. 
Let $d(x, y)$ and $d_{\rho}(x, y)$ denote the distances between $x$ and $y$ with respect to $d s^{2}$ and $\rho^{2} d s^{2}$, respectively; $B(x, R)=\{y \in M ; d(x, y)<R\}$ and $B_{\rho}(x, R)=\left\{y \in M ; d_{\rho}(x, y)<R\right\}$. For a fixed point $p \in M$, we denote $r(x)$ and $r_{\rho}(x)$ the distance functions with respect to metric $d s^{2}$ and conformal metric $\rho d s^{2}$ from $p$, respectively; $B(R)=\{x \in M ; r(x)<R\}$ and $B_{\rho}(R)=$ $\left\{x \in M ; r_{\rho}(x)<R\right\}$.

We need the following construction of harmonic functions [13, §5].

Suppose $M$ has at least two non-parabolic ends $E_{1}$ and $E_{2}$. A theory of $\mathrm{Li}$ and Tam [10] asserts that one can get a non-constant bounded harmonic function $f$ with finite Dirichlet integral by taking a convergent subsequence of the harmonic functions $f_{R}$ as $R \rightarrow+\infty$, satisfying

$$
\Delta f_{R}=0 \quad \text { on } B(R),
$$

with boundary conditions

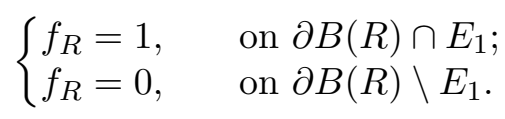

It follows from the maximum principle that $0 \leq f_{R} \leq 1$ for all $R$ and hence $0 \leq f \leq 1$.

Now we prove Theorem 1.2.

Proof. If $M$ has at least two non-parabolic ends, then there exists a bounded harmonic function $f$ with finite Dirichlet integral constructed as above. We may assume that inf $f=0$ and $\sup f=1$.

Then the Bochner formula and the lower bound of the Ricci curvature imply (cf. [13, Lemm 4.1])

$$
\Delta|\nabla f| \geq-(n-1) \tau|\nabla f|+\frac{1}{n-1} \frac{\left.|\nabla| \nabla f\right|^{2}}{|\nabla f|} .
$$

Set $\alpha=\frac{n-2}{n-1}$ and $g=|\nabla f|^{\alpha}$. Equation (2.1) implies

$$
\begin{aligned}
\Delta g & =\left.\alpha(\alpha-1)|\nabla f|^{\alpha-2}|\nabla| \nabla f\right|^{2}+\alpha|\nabla f|^{\alpha-1} \Delta|\nabla f| \\
& \geq-(n-2) \tau g .
\end{aligned}
$$


We will show inequality (2.2) is actually an equality. For any nonnegative compactly supported piecewise smooth function $\phi$ on $M$, we have

$$
\begin{aligned}
\int_{M} & \phi^{2} g(\Delta g+(n-2) \tau g) \\
& =-2 \int_{M} \phi g\langle\nabla g, \nabla \phi\rangle-\int_{M} \phi^{2}|\nabla g|^{2}+\int_{M}(n-2) \tau(\phi g)^{2} \\
& \leq-2 \int_{M} \phi g\langle\nabla g, \nabla \phi\rangle-\int_{M} \phi^{2}|\nabla g|^{2}+\int_{M}|\nabla(\phi g)|^{2} \\
& =\int_{M}|\nabla \phi|^{2}|\nabla f|^{2(n-2) /(n-1)}=\int_{M}|\nabla \phi|^{2} g^{2} .
\end{aligned}
$$

The inequality in (2.3) holds since $\tau$ satisfies Poincaré inequality.

Choose $\phi=\psi \chi$, where $\psi$ and $\chi$ will be chosen later and $\psi$ denotes a piecewise smooth compactly supported function on $M$. Then

$$
\begin{aligned}
\int_{M}|\nabla \phi|^{2} g^{2} \leq & 2 \int_{M}|\nabla \psi|^{2} \chi^{2}|\nabla f|^{2(n-2) /(n-1)} \\
& +2 \int_{M}|\nabla \chi|^{2} \psi^{2}|\nabla f|^{2(n-2) /(n-1)}
\end{aligned}
$$

We first consider the case of $n \geq 4$. For $R>1$, we let $\psi(x)$ be a function depending on the $\rho$-distance:

$$
\psi(x)= \begin{cases}1 & \text { on } B_{\rho}(R-1), \\ R-r_{\rho} & \text { on } B_{\rho}(R) \backslash B_{\rho}(R-1), \\ 0 & \text { on } M \backslash B_{\rho}(R) .\end{cases}
$$

For $\sigma \in(0,1)$ and $\epsilon \in\left(0, \frac{1}{2}\right)$, we define $\chi$ on the level sets of $f$ :

$$
\chi(x)= \begin{cases}0 & \text { on } \mathcal{L}(0, \sigma \epsilon) \cup \mathcal{L}(1-\sigma \epsilon, 1) \\ (\epsilon-\sigma \epsilon)^{-1}(f-\sigma \epsilon) & \text { on } \mathcal{L}(\sigma \epsilon, \epsilon) \cap\left(M \backslash E_{1}\right) \\ (\epsilon-\sigma \epsilon)^{-1}(1-\sigma \epsilon-f) & \text { on } \mathcal{L}(1-\epsilon, 1-\sigma \epsilon) \cap E_{1} \\ 1 & \text { otherwise }\end{cases}
$$

where we denote the set $\mathcal{L}(a, b)=\{x \in M \mid a<f(x)<b\}$.

Denote the set

$$
\Omega=E_{1} \cap\left(B_{\rho}(R) \backslash B_{\rho}(R-1)\right) \cap(\mathcal{L}(\sigma \epsilon, 1-\sigma \epsilon)) .
$$


Recall the growth estimate for $|\nabla f|[13$, Corollary 2.3]:

$$
\int_{B_{\rho}(R+1) \backslash B_{\rho}(R)}|\nabla f|^{2} \leq C e^{-2 R},
$$

and the decay estimate for $f[13,(2.10)]$ :

$$
\int_{E_{1} \cap B_{\rho}(R+1) \backslash E_{1} \cap B_{\rho}(R)} \rho(1-f)^{2} \leq C e^{-2 R} .
$$

We have

$$
\left(\int_{\Omega}|\nabla f|^{2}\right)^{(n-2) /(n-1)} \leq C e^{-(2(n-2) /(n-1)) R}
$$

and with notation $S(R)$ as in the statement of theorem,

$$
\begin{aligned}
\int_{\Omega} \rho^{n-1} & \leq(S(R))^{2(n-2)} \int_{\Omega} \rho \\
& \leq(\sigma \epsilon)^{-2}(S(R))^{2(n-2)} \int_{\Omega} \rho(1-f)^{2} \\
& \leq C(S(R))^{2(n-2)}(\sigma \epsilon)^{-2} e^{-2 R} .
\end{aligned}
$$

Hence, by $\left|\nabla r_{\rho}\right|(x)=\rho(x),(2.5)$ and (2.6), we have

$$
\begin{aligned}
\int_{E_{1}} & |\nabla \psi|^{2} \chi^{2}|\nabla f|^{2(n-2) /(n-1)} \\
\leq & \int_{\Omega} \rho|\nabla f|^{2(n-2) /(n-1)} \\
\leq & \left(\int_{\Omega}|\nabla f|^{2}\right)^{(n-2) /(n-1)}\left(\int_{\Omega} \rho^{n-1}\right)^{1 /(n-1)} \\
\leq & C(\sigma \epsilon)^{-2 /(n-1)}(S(R))^{2(n-2) /(n-1)} e^{-2((n-2) /(n-1)) R-(2 /(n-1)) R} \\
\leq & C(\sigma \epsilon)^{-2 /(n-1)}(S(R))^{2(n-2) /(n-1)} e^{-2 R} .
\end{aligned}
$$

Note the assumption that the Ricci curvature of $M$ is bounded from below by $-(n-1) \tau(x)$. Then the local gradient estimate of Cheng and Yau [2] (cf. [12]) implies that there exists a constant $C_{n}$ depending on $n$ such that

$$
|\nabla f|(x) \leq C_{n}\left(\sup _{y \in B(x, R)} \sqrt{\tau(y)}+R^{-1}\right)|f(x)|, \quad x \in M,
$$

for all $R>0$. 
Set $\bar{\rho}(x)=\frac{1}{2} \rho(x)+\frac{1}{2}(n-2) \tau(x), x \in M$. Then $\sqrt{\tau} \leq \sqrt{\frac{2}{n-2} \bar{\rho}}$ and

$$
|\nabla f|(x) \leq C\left(\sup _{y \in B(x, R)} \sqrt{\bar{\rho}}(y)+R^{-1}\right)|f(x)|
$$

Fix $x \in M$ and consider the function $\eta(R)=\sqrt{2} R-\left(\sup _{B(x, R)} \sqrt{\bar{\rho}}\right)^{-1}$. Observe that $\eta(R)$ tends to $+\infty$ as $R \rightarrow \infty$ and tends to a negative number as $R \rightarrow 0$. There exists a $R_{0}$ depending on $x$ such that $\sqrt{2} R_{0}=\left(\sup _{B\left(x, R_{0}\right)}\right.$ $\sqrt{\bar{\rho}})^{-1}$. Hence,

$$
|\nabla f|(x) \leq C\left(\sup _{B\left(x, R_{0}\right)} \sqrt{\bar{\rho}}\right)|f(x)| .
$$

For any $y \in B\left(x, R_{0}\right)$, let $\gamma(s), s \in[0, l]$ be a minimizing geodesic connecting $x$ and $y$ with respect to the background metric $d s^{2}$, where $s$ is the arc-length of $\gamma$ in $d s^{2}$. The distance $d_{\rho}(x, y)$ with respect to $\rho d s^{2}$ satisfies

$$
\begin{aligned}
d_{\rho}(x, y) & \leq \int_{0}^{l} \sqrt{\rho(\gamma(s))} d s \\
& \leq \int_{0}^{l} \sqrt{2} \sqrt{\bar{\rho}(\gamma(s))} d s \\
& \leq\left(\sup _{B\left(x, R_{0}\right)} \sqrt{\bar{\rho}}\right)\left(\sqrt{2} R_{0}\right)=1
\end{aligned}
$$

This implies $B\left(x, R_{0}\right) \subset B_{\rho}(x, 1)$. Hence,

$$
|\nabla f|(x) \leq C\left(\sup _{B_{\rho}(x, 1)} \sqrt{\bar{\rho}}\right)|f(x)|, \quad x \in M .
$$

Similarly, we have

$$
|\nabla f|(x) \leq C\left(\sup _{B_{\rho}(x, 1)} \sqrt{\bar{\rho}}\right)|1-f(x)|, \quad x \in M .
$$


On $E_{1}$, we have

$$
\begin{aligned}
& \int_{E_{1}}|\nabla \chi|^{2} \psi^{2}|\nabla f|^{2(n-2) /(n-1)} \\
& \leq C((1-\sigma) \epsilon)^{-2} \int_{\mathcal{L}(1-\epsilon, 1-\sigma \epsilon) \cap E_{1} \cap B_{\rho}(R)}|\nabla f|^{2(n-2) /(n-1)+2} \\
& \leq C(S(R+1))^{2(n-2) /(n-1)}((1-\sigma) \epsilon)^{-2} \\
& \quad \times \int_{\mathcal{L}(1-\epsilon, 1-\sigma \epsilon) \cap E_{1} \cap B_{\rho}(R)}|\nabla f|^{2}(1-f)^{2(n-2) /(n-1)} .
\end{aligned}
$$

Note that [13, Lemma 5.1] asserts that the integral of $|\nabla f|$ on the level set $l(t)=\{x \in M \mid f(x)=t\}, 0 \leq t \leq 1$, is independent of $t$. Using this conclusion and the co-area formula and [13, Lemma 5.1], we have

$$
\begin{aligned}
\int_{\mathcal{L}(1-\epsilon, 1-\sigma \epsilon) \cap E_{1} \cap B_{\rho}(R)}|\nabla f|^{2}(1-f)^{2(n-2) /(n-1)} \\
\leq \int_{1-\epsilon}^{1-\sigma \epsilon}(1-t)^{2(n-2) /(n-1)} \int_{l(t) \cap E_{1} \cap B_{\rho}(R)}|\nabla f| d A d t \\
\leq C \int_{l(b)}|\nabla f| d A \int_{1-\epsilon}^{1-\sigma \epsilon}(1-t)^{2(n-2) /(n-1)} d t \\
=C \int_{l(b)}|\nabla f| d A \cdot\left(1-\sigma^{2(n-2) /(n-1)+1}\right) \epsilon^{2(n-2) /(n-1)+1}
\end{aligned}
$$

Substitute (2.14) into (2.13). Then

$$
\begin{aligned}
\int_{E_{1}}|\nabla \chi|^{2} \psi^{2}|\nabla f|^{2(n-2) /(n-1)} \leq & C(S(R+1))^{2(n-2) /(n-1)}(1-\sigma)^{-2} \\
& \times\left(1-\sigma^{2(n-2) /(n-1)+1}\right) \epsilon^{(n-3) /(n-1)}
\end{aligned}
$$

Setting $\sigma=\frac{1}{2}$, we have

$$
\begin{aligned}
\int_{E_{1}}|\nabla \phi|^{2}|\nabla f|^{2(n-2) /(n-1)} \leq & C(S(R+1))^{2(n-2) /(n-1)} \\
& \times\left(e^{-2 R} \epsilon^{-2 /(n-1)}+\epsilon^{(n-3) /(n-1)}\right) .
\end{aligned}
$$


Let us choose $\epsilon=e^{-2 R}$. Then

$$
\int_{E_{1}}|\nabla \phi|^{2}|\nabla f|^{2(n-2) /(n-1)} \leq C(S(R+1))^{2(n-2) /(n-1)} e^{-2((n-3) /(n-1)) R} .
$$

Using $f$ instead of $1-f$, similar to the above argument, we have that on $M \backslash E_{1}$,

$$
\int_{M \backslash E_{1}}|\nabla \phi|^{2}|\nabla f|^{2(n-2) /(n-1)} \leq C(S(R+1))^{2(n-2) /(n-1)} e^{-2((n-3) /(n-1)) R} .
$$

Hence,

$$
\int_{M}|\nabla \phi|^{2}|\nabla f|^{2(n-2) /(n-1)} \leq C(S(R+1))^{2(n-2) /(n-1)} e^{-2((n-3) /(n-1)) R} .
$$

Let $R \rightarrow+\infty$. By the assumption on $S(R)$, the left in $(2.18)$ is identically zero. By (2.3), we conclude that $(2.2)$ is actually an equality and hence the improved Bochner inequality (2.1) must be an equality. Note that [13, Lemma 4.1] asserts that if equality in inequality (2.1) holds, the metric of $M$ must be a warped product as described in the theorem. We obtain the conclusion of theorem in the case of $n \geq 4$.

In the case of $n=3$, we may choose $\psi$ as above and $\chi$ to be

$$
\chi(x)= \begin{cases}0 & \text { on } \mathcal{L}(0, \sigma \epsilon) \cup \mathcal{L}(1-\sigma \epsilon, 1), \\ (-\log \sigma)^{-1}(\log f-\log (\sigma \epsilon)) & \text { on } \mathcal{L}(\sigma \epsilon, \epsilon) \cap\left(M \backslash E_{1}\right), \\ (-\log \sigma)^{-1}(\log (1-f)-\log (1-\sigma \epsilon)) & \text { on } \mathcal{L}(1-\epsilon, 1-\sigma \epsilon) \cap E_{1} \\ 1 & \text { otherwise. }\end{cases}
$$

By an argument similar to the above one for $n \geq 4$ (combining with the corresponding estimates for $n=3$ in [13, Theorem 5.2]), we have the estimate

$$
\int_{M}|\nabla \phi|^{2}|\nabla f|^{2(n-2) /(n-1)} \leq C S(R+1)\left(\sigma^{-1} \epsilon^{-1} e^{-2 R}+(-\log \sigma)^{-1}\right)
$$

Choose $\sigma=\epsilon=e^{-R q(R)}$ with $q(R)=\sqrt{\frac{S(R+1)}{R}}$. Then using the argument in [13], we have the right side of (2.19) tends to zero as $R \rightarrow+\infty$. We conclude that (2.2) is actually an equality and hence the theorem holds for $n=3$. 


\section{Application to minimal hypersurfaces}

Let $M^{n}$ be a complete minimal hypersurface in $\mathbb{R}^{n+1}$ for $n \geq 3$. We first give some examples of the metric $\rho d s^{2}$ such that $M$ satisfies property $\left(\mathcal{P}_{\rho}\right)$.

Example 3.1. Let $\bar{d}(x, y), x, y \in \mathbb{R}^{n+1}$, denote the distance between $x$ and $y$ in $\mathbb{R}^{n+1}$. Denote by $\bar{r}(x), x \in M$, the extrinsic distance function $\bar{d}(x, o)$ from a fixed point $o \in \mathbb{R}^{n+1}$ ( $o$ may or may not be in $M$ ). It is known that

$$
\Delta \bar{r} \geq(n-1) \bar{r}^{-1},
$$

where $\Delta$ is the Laplacian on $M$.

For any $\phi \in C_{o}^{\infty}(M)$,

$$
\begin{gathered}
(n-1) \int_{M} \bar{r}^{-2} \phi^{2} \leq \int_{M} \bar{r}^{-1} \phi^{2} \Delta \bar{r} \\
=-2 \int_{M} \bar{r}^{-1} \phi\langle\nabla \phi, \nabla \bar{r}\rangle+\int_{M} \bar{r}^{-2} \phi^{2}|\nabla \bar{r}|^{2} \\
\leq 2 \int_{M} \bar{r}^{-1} \phi|\nabla \phi|+\int_{M} \bar{r}^{-2} \phi^{2} . \\
(n-2) \int_{M} \bar{r}^{-2} \phi^{2} \leq 2 \int_{M} \bar{r}^{-1} \phi|\nabla \phi| \\
\leq 2\left(\int_{M} \bar{r}^{-2} \phi^{2}\right)^{1 / 2}\left(\int_{M}|\nabla \phi|^{2}\right)^{1 / 2}
\end{gathered}
$$

Hence,

$$
\int_{M}|\nabla \phi|^{2} \geq \frac{(n-2)^{2}}{4} \int_{M} \bar{r}^{-2} \phi^{2} \quad \text { for all } \quad \phi \in C_{o}^{+\infty}(M) .
$$

Let $\rho(x)=\frac{(n-2)^{2}}{4} \bar{r}^{-2}(x), x \in M$. Inequality (3.1) asserts the Poincaré inequality holds with weight function $\rho$.

Further the metric $\rho d s^{2}$ is complete. Indeed, take a fixed point $p \in M$ with $p \neq o$. Let $r(x), x \in M$, denote the intrinsic distance from $p$. Note that $\bar{r}(x) \leq \bar{d}(o, p)+\bar{d}(x, p) \leq r_{0}+r(x)$, where $r_{0}=\bar{d}(o, p)>0$. Then $\bar{r}^{-2}(x)>$ $\left(r_{0}+r(x)\right)^{-2}$. It is known that the metric $\left(r_{0}+r(x)\right)^{-2} d s^{2}$ is complete. Hence $\rho d s^{2}$ is complete.

Thus we obtain that $M$ has property $\left(\mathcal{P}_{\rho}\right)$ for $\rho$. 
Example 3.2. Using smoothing technique, we may modify $\rho=\frac{(n-2)^{2}}{4} \bar{r}^{-2}$ in Example 3.1 to get a bounded smooth positive function $\rho_{1}(x)=\rho_{1}(\bar{r}(x))$, $x \in M$, such that $M$ has property $\left(\mathcal{P}_{\rho}\right)$ for $\rho_{1}$.

Indeed, let positive number $0<b \leq r_{0}$ be fixed, we can choose number $a, 0<a<b$, such that function $\zeta(\bar{r})=\frac{(n-2)^{2}}{4}\left(\bar{r}^{-2}-e^{-1 /(\bar{r}-b)^{2}}\right)$ is strictly decreasing in $(a, b)$ as $\bar{r}$ tends increasingly to $b$ and construct the smooth $\rho_{1}$

$$
\rho_{1}(\bar{r}(x))= \begin{cases}h(\bar{r}) & \text { for } \bar{r}(x) \leq a \\ \zeta(\bar{r}) & \text { for } a<\bar{r}(x)<b \\ \rho(\bar{r}) & \text { for } \bar{r}(x) \geq b\end{cases}
$$

where $h(\bar{r})$ is chosen to be bounded and to satisfy $\rho(\bar{r}) \geq h(\bar{r}) \geq \rho(b)$ for $\bar{r} \leq a$.

Observe that $\rho_{1} \leq \rho$. Hence the Poincaré inequality holds for $\rho_{1}$. Moreover, $\rho_{1} d s^{2}$ is complete since $\rho_{1}(x) \geq \frac{(n-2)^{2}}{4}\left(r_{0}+r(x)\right)^{-2}$. In fact, for $\bar{r}(x) \geq$ $b, \rho_{1}=\rho$. Note that for $\bar{r}(x)<b, \rho_{1}(\bar{r}(x)) \geq \rho(b)$ and $0<b \leq r_{0}$. Hence, $\rho_{1}(\bar{r}(x)) \geq \frac{(n-2)^{2}}{4}\left(r_{0}+r(x)\right)^{-2}$ for $\bar{r}(x)<b$.

Example 3.3. Under the above notations, choose $\rho_{2}(x)=\frac{(n-2)^{2}}{4}\left(r_{0}\right.$ $+r(x))^{-2}, x \in M$. Since $\rho_{2} \leq \rho$, Poincaré inequality holds with weight function $\rho_{2}$. By the completeness of the metric $\rho_{2} d s^{2}$, we know $M$ has property $\left(\mathcal{P}_{\rho}\right)$ for $\rho_{2}$.

Theorem 3.1 (Theorem 1.1). Let $M$ be an $\frac{n-2}{n}$-stable complete minimal hypersurface in $\mathbb{R}^{n+1}, n \geq 3$, and the norm of its second fundamental form satisfies

$$
\begin{aligned}
& \lim _{R \rightarrow+\infty} \sup _{B(R)}|A| / R^{(n-3) / 2}=0, \quad \text { for } \quad n \geq 4 ; \\
& \lim _{R \rightarrow+\infty} \sup _{B(R)}|A| / \ln R=0, \quad \text { for } \quad n=3,
\end{aligned}
$$

then $M$ either has one end or must be a catenoid.

Proof. For any point $q \in M$ and any unit tangent vector $v \in T_{q} M$, we can choose an orthonormal frame $\left\{e_{1}, e_{2}, \ldots, e_{n}\right\}$ on $M$ at $q$ such that $e_{1}=v$. 
From the Gauss equation, we have

$$
\begin{aligned}
|A|^{2} & \geq h_{11}^{2}+\sum_{i=2}^{n} h_{i i}^{2}+2 \sum_{i=1}^{n} h_{1 i}^{2} \\
& \geq h_{11}^{2}+\frac{\left(\sum_{i=2}^{n} h_{i i}\right)^{2}}{n-1}+2 \sum_{i=1}^{n} h_{1 i}^{2} \\
& \geq \frac{n}{n-1}\left(h_{11}^{2}+\sum_{i=2}^{n} h_{1 i}^{2}\right) \\
& \geq-\frac{n}{n-1} \operatorname{Ric}_{M}\left(e_{1}, e_{1}\right) .
\end{aligned}
$$

Then $\operatorname{Ric}_{M}(v, v) \geq-\frac{n-1}{n}|A|^{2}$.

Let us choose $\tau=\frac{|A|^{2}}{n}$ and $\rho=\rho_{1}$ (or $\rho_{2}$ ) in Theorem 1.2. By the boundedness of $\rho_{1}$ (or $\rho_{2}$ ), the growth assumption (1.4) on $\rho$ is satisfied. Now we will assert that the growth assumption (1.5) on $\tau$ is also satisfied.

It can be verified directly that a minimizing geodesic starting from the fixed point $p$ with respect to $d s^{2}$ is also a minimizing geodesic starting from $p$ with respect to $\rho_{2} d s^{2}$. Then by direct calculation, we have $B_{\rho_{2}}(\bar{R})=B(R)$, where $\bar{R}=\frac{n-2}{2} \ln \left(1+\frac{R}{r_{0}}\right)$. Then for $n \geq 4$

$$
\lim _{\bar{R} \rightarrow+\infty} \sup _{B_{\rho_{2}}(\bar{R})}|A| e^{-(n-3) /(n-2) \bar{R}}=C \lim _{R \rightarrow+\infty} \sup _{B(R)}|A| R^{-(n-3) / 2}=0
$$

For $n=3$,

$$
\lim _{\bar{R} \rightarrow+\infty} \sup _{B_{\rho_{2}}(\bar{R})}|A| \bar{R}^{-1}=C \lim _{R \rightarrow+\infty} \sup _{B(R)}|A|(\ln R)^{-1}=0 .
$$

If $\rho=\rho_{1}$, by $\rho_{1} \geq \rho_{2}, B_{\rho_{1}}(\bar{R}) \subseteq B_{\rho_{2}}(\bar{R})$ and hence the growth assumption on $\tau$ also holds for $\rho_{1}$.

Therefore, the conclusion of Theorem 1.2 is valid. Let us assume that $M$ has at least two ends. Since every end of a complete non-compact minimal hypersurface in $\mathbb{R}^{n+1}$ is non-parabolic ([1], see its proof also in [4]), by Theorem 1.2, we know that $M$ has exactly two non-parabolic ends and $M=\mathbb{R} \times N$ with the warped product metric

$$
d s_{M}^{2}=d t^{2}+\eta^{2}(t) d s_{N}^{2}
$$


for some compact manifold $N$ and some positive function $\eta(t)$. Moreover, $|A|$ is a function of $t$ alone satisfying

$$
(n-2) \eta^{\prime \prime} \eta^{-1}=\frac{|A|}{n}
$$

Hence, $M$ has a rotationally symmetric metric. By a result of do Carmo and Dajczer [5, Corollary 4.4], it implies that every part of $M$ is a part of a catenoid. Hence $M$ is contained in a catenoid $\mathcal{C}$ by minimality of the immersion. Since $M$ is complete and the catenoid $\mathcal{C}$ is simply connected because $n \geq 3, M$ must be an embedded hypersurface, see [15, p. 330]. Hence $M$ is the catenoid.

Theorem 3.1 implies directly that

Corollary 3.1 (Corollary 1.1). Let $M$ be an $\frac{n-2}{n}$-stable complete minimal hypersurface in $\mathbb{R}^{n+1}, n \geq 3$, with at least two ends. If its second fundamental form is bounded, then $M$ must be a catenoid.

\section{Acknowledgment}

The authors would like to thank Jiaping Wang for very helpful discussions during their visit to the Chinese Hong Kong University. Both authors are partially supported by $\mathrm{CNPq}$ and Faperj of Brazil.

\section{References}

[1] H. Cao, Y. Shen and S. Zhu, The structure of stable minimal hypersurfaces in $\mathbb{R}^{n+1}$, Math. Res. Lett. 4 (1997), 637-644.

[2] S.Y. Cheng and S.-T. Yau, Differential equations on Riemannian manifolds and their geometric applications, Comm. Pure Appl. Math. 28 (1975), 333-354.

[3] X. Cheng, One end theorem and application to stable minimal hypersurfaces, Arch Math. 90 (2008), 461-470.

[4] X. Cheng, L.F. Cheung and D. Zhou, The structure of weakly stable constant mean curvature hypersurfaces, Tohoku Math. J. 60 (2008), 101-121.

[5] M. do Carmo and M. Dajczer, Rotation hypersurface in spaces of constant curvature, Trans. Amer. Math. Soc. 277 (1983), 685-709. 
[6] M. do Carmo and C.K. Peng, Stable complete minimal surfaces in $\mathbb{R}^{3}$ are planes, Bull. Amer. Math. Soc. 1 (1979), 903-906.

[7] D. Fischer-Colbrie and R. Schoen, The structure of complete stable minimal surfaces in 3-manifolds of nonnegative scalar curvature, Comm. Pure Appl. Math. 33 (1980), 199-211.

[8] S. Kawai, Operator $\Delta-a K$ on surfaces, Hokkaido Math. J. 17 (1988), $147-150$.

[9] F. Lopez and A. Ros, Complete minimal surfaces with index one and stable constant mean curvature surfaces, Comment. Math. Helv. 64 (1989), 34-43.

[10] P. Li and L.F. Tam, Harmonic functions and the structure of complete manifolds, J. Diff. Geom. 35 (1992), 359-383.

[11] P. Li and J. Wang, Complete manifolds with positive spectrum, J. Diff. Geom. 58 (2001), 501-534.

[12] - Complete manifolds with positive spectrum II, J. Diff. Geom. 62 (2002), 143-162.

[13] P. Li and J. Wang, Weighted Poincaré inequality and rigidity of complete manifolds, Ann. Sci. Éc. Norm. Sup. 39 (2006), 921-982.

[14] W. Meeks III, J. Pérez and A. Ros, Liouville-type properties for embedded minimal surfaces, Comm. Anal. Geom. 14 (2006), 703-723.

[15] M. Spivak, A Comprehensive introduction to differential geometry, Vol. 4, Publish or Perish (1970-75).

[16] L.F. Tam and D. Zhou, Stability properties for the higher dimensional catenoid in $\mathbb{R}^{n+1}$, arXiv:0708.3310v1.

Insitituto DE MATEMÁtica

Universidade Federal Fluminense - UFF Centro

Niterói

RJ 24020-140

BRAZIL

E-mail address: xcheng@impa.br; zhou@impa.br

Received August 8, 2008 